\title{
(2) OPEN ACCESS \\ Anti-VEGF-resistant subretinal fluid is associated with better vision and reduced risk of macular atrophy
}

\author{
Marco A Zarbin @ (1), Lauren Hill, ${ }^{2}$ Andreas Maunz, ${ }^{3}$ Martin Gliem, ${ }^{3}$ Ivaylo Stoilov ${ }^{2}$
}

\begin{abstract}
- Additional supplemental material is published online only. To view, please visit the journal online (http://dx.doi. org/10.1136/bjophthalmol2020-318688).
\end{abstract}

${ }^{1}$ Institute of Ophthalmology and Visual Science, Rutgers New Jersey Medical School, Newark, New Jersey, USA

${ }^{2}$ Medical Affairs Ophthalmology, Genentech, Inc, South San Francisco, California, USA ${ }^{3}$ Roche Pharma Research and Early Development, Basel, Switzerland

\section{Correspondence to} Dr Marco A Zarbin, Institute of Ophthalmology and Visual Science, Rutgers New Jersey Medical School, Newark, New Jersey, USA; zarbin@rutgers.edu

Received 22 December 2020 Revised 7 April 2021

Accepted 30 April 2021
D) Check for updates

(C) Author(s) (or their employer(s)) 2021. Re-use permitted under CC BY-NC. No commercial re-use. See rights and permissions. Published by BMJ.

To cite: Zarbin MA, Hill L, Maunz A, et al.

Br J Ophthalmol Epub ahead of print: [please include Day Month Year]. doi:10.1136/

bjophthalmol-2020-318688

\section{ABSTRACT}

Background/aim To evaluate relationships between subretinal fluid (SRF), macular atrophy (MA) and visual outcomes in ranibizumab-treated neovascular agerelated macular degeneration (nAMD).

Methods This post hoc HARBOR trial (NCT00891735) analysis included ranibizumab-treated $(0.5$ or $2.0 \mathrm{mg}$, monthly or as-needed, all treatment arms pooled) eyes with nAMD and baseline (screening, baseline and week 1) SRF. SRF presence, SRF thickness $(0,>0-50$, $>50-100$ and $>100 \mu \mathrm{m}$ ) and subretinal fluid volume (SRFV) were determined by spectral domain optical coherence tomography (SD-OCT). Best-corrected visual acuity (BCVA) was assessed. MA was identified using fluorescein angiograms and colour fundus photographs, as well as SD-OCT.

Results Seven hundred eighty-five of 1097 eyes met analysis criteria. In eyes without baseline MA, residual versus no SRF at month (M) 3 was associated with lower MA rates at M12 (5.1\% vs $22.1 \%)$ and $\mathrm{M} 24(13.3 \%$ vs $31.2 \%$ ) (both $p<0.0001$ ); MA percentages at M12/M24 were similar among patients with residual SRF at M6. Higher baseline SRFV was associated with a lower MA rate. Greater mean BCVA was observed with residual SRF of any thickness ( $>0-50 \mu \mathrm{m}, 71.2$ letters; $>50-100 \mu \mathrm{m}$, 71.3 letters; > $100 \mu \mathrm{m}, 69.2$ letters) versus no SRF (63.6 letters), but the change in BCVA from baseline to $\mathrm{M} 12$ or M24 was the same for eyes with or without treatmentresistant subretinal fluid (TR-SRF) at M3 or M6.

Conclusion TR-SRF was not detrimental to vision outcomes over 2 years, regardless of thickness. MA rates were significantly higher without TR-SRF.

\section{INTRODUCTION}

The pivotal ANCHOR and MARINA trials established the effectiveness of monthly intravitreal ranibizumab injections for the treatment of neovascular age-related macular degeneration (nAMD). ${ }^{12}$ The uniform monthly regimen, however, placed a significant treatment burden on patients and physicians. The PrONTO study demonstrated that optical coherence tomography (OCT)-guided treatment can reduce the number of injections while achieving vision outcomes comparable with those obtained with monthly dosing. ${ }^{3}$ This paradigm, known as a pro re nata (PRN, as-needed) regimen, later evolved into treat-and-extend posology. ${ }^{4} 5$ With OCT becoming the main tool to assess disease activity, the success of anti-vascular endothelial growth factor (VEGF) treatment in individual patients evolved to be judged principally by the resolution of subretinal fluid (SRF) and intraretinal fluid
(IRF) and the flattening of pigment epithelial detachments. Data from subsequent randomised clinical trials, however, have demonstrated that nAMD eyes with anti-VEGF-resistant SRF (ie, SRF that persists despite aggressive anti-VEGF therapy) tend to have better visual outcomes than those with complete resolution of SRF or IRF. $^{6-8}$ In contrast, the presence of anti-VEGFresistant IRF is associated with worse long-term visual outcomes, which may reflect loss of integrity of the external limiting membrane associated with more severe retinal damage. ${ }^{9}{ }^{10}$ To understand whether SRF has a biphasic effect (ie, some SRF is good, but a lot of SRF is bad), we decided to investigate the impact of different amounts of residual SRF (at month (M) 3 and M6, in 50- $\mathrm{m}$ increments) as well as baseline subretinal fluid volume (SRFV) on vision outcomes and the rate of developing macular atrophy (MA) in the HARBOR trial.

\section{MATERIALS AND METHODS}

The study design of the phase III HARBOR trial (NCT00891735) of ranibizumab in treatmentnaive patients with subfoveal nAMD is shown in online supplemental figure $\mathrm{S} 1$. The trial has been described in detail previously. ${ }^{11}{ }^{12}$ Briefly, trial participants were randomised to receive ranibizumab 0.5 or $2.0 \mathrm{mg}$, monthly or PRN, after three loading doses. The primary endpoint was mean change from baseline in best-corrected visual acuity (BCVA) at M12. ${ }^{11}$ Retina anatomy was evaluated with spectral domain optical coherence tomography (SD-OCT). All groups continued on the same treatment schedule through M24 (online supplemental figure S1). ${ }^{12}$

For this post hoc analysis, all ranibizumab treatment arms were pooled, and analysis was limited to eyes with SRF at screening, baseline or week $1(n=785)$. Of the 1097 total patients in the HARBOR trial, there were 917 evaluable studies, among which 785 (85.6\%) patients had SRF present at screening, baseline or week 1 . Thus, 312 patients were excluded from the 1097 total patients in the HARBOR trial. SRF presence and thickness were evaluated using SD-OCT. SRF, defined as exudation occurring between the photoreceptor layer and the retinal pigment epithelium (RPE), was determined from all SD-OCT b-scans for each patient by two masked graders. A third grader adjudicated any discrepancies between the first two assessments. SRF thickness was measured at the two furthest vertical points of the SRF. Eyes were grouped according to SRF thickness $(0,>0-50,>50-100$ or $>100 \mu \mathrm{m})$. 
SRFV was evaluated at baseline using a deep learning algorithm (convolutional neural network). For this purpose, a sparse selection of $19 \mathrm{~b}$-scans per volume across a total of 50 volumes (950 b-scans in total) obtained from the Cirrus (Carl Zeiss Meditec) OCT scans used in HARBOR was annotated by experts from the Liverpool Ophthalmic Reading Centre by drawing contours of SRF. Subsequently, the U-Net, a convolutional neural network for biomedical image segmentation, was trained to recognise SRF using the annotated volumes as training material. ${ }^{13}$ Then, all OCT scans of HARBOR were segmented using the resulting model, reaching a Sørensen-Dice similarity coefficient score of 0.6466 (SD \pm 0.1738 , $n=33$ ) on the holdout set. The segmentations should be considered proof of concept, but they showed promise to deliver value despite the very early version. BCVA was assessed using standard ETDRS protocols. SRFV was evaluated in circles centred under the fovea, with diameters of 1000, 3000 and $6000 \mu \mathrm{m}$ (hereon listed as 1, 3 and $6 \mathrm{~mm}$ ).

MA presence was determined in two ways. First, MA presence was identified using fluorescein angiograms (FAs) and colour fundus photographs (CFPs) by masked graders. MA was defined as sharply demarcated areas of RPE depigmentation with increased visibility of choroidal vessels through the lesion on CFPs or FA, $\geq 250-\mu \mathrm{m}$ diameter, corresponding to flat areas of well-demarcated staining on FA, and included all atrophy immediately within, adjacent and non-adjacent to choroidal neovascularisation (CNV) lesions (active or regressed). ${ }^{7}$ Second, MA presence was identified using SD-OCT images that were reread using Zeiss-Cirrus HD-OCT software, as described in detail by Sadda et al. ${ }^{14}$ Briefly, images were graded by two independent unmasked graders. The first grader reviewed, and if this grader required review of a case, then a second independent unmasked grader reviewed the case. If there was non-consensus in answers between the first and second graders that could not be successfully adjudicated, then a review by the reading centre principal investigator was triggered.

\section{Statistical analyses}

Descriptive statistics were used to evaluate differences in vision outcomes by SRF thickness and presence of MA by fluid status. For continuous variables, means, 95\% CIs and Student's t-test $\mathrm{p}$ values

\begin{tabular}{ll}
\hline $\begin{array}{l}\text { Table } 1 \text { Summary of key patient demographics and baseline } \\
\text { characteristics }\end{array}$ \\
\hline Characteristic & $\begin{array}{l}\text { Pooled treatment group } \\
(\mathbf{n}=785)\end{array}$ \\
\hline Age (years), mean (SD) & $77.5(8.2)$ \\
\hline Female, $\mathrm{n}(\%)$ & $449(57.2)$ \\
White, $\mathrm{n}(\%)$ & $760(96.8)$ \\
\hline Hispanic or Latino ethnicity, $\mathrm{n}(\%)$ & $23(2.9)$ \\
\hline BCVA (ETDRS letters), mean (SD) & $54.6(12.5)$ \\
\hline Presence of MA, $\mathrm{n}(\%)^{*}$ & $63(8.0)$ \\
\hline SRF thickness at baseline ( $\mu \mathrm{m}), \mathrm{n}(\%) \dagger$ & $49(6.7)$ \\
\hline$>0-50$ & $189(25.7)$ \\
$>50-100$ & $498(67.7)$ \\
\hline$>100$ &
\end{tabular}

Key demographics and baseline characteristics for ranibizumab-treated patients from the HARBOR trial with SRF at baseline (defined as screening, baseline or week 1). Patients with SRF at baseline in all treatment groups were pooled in this post hoc analysis.

*Forty-six patients had an MA status at baseline that could not be determined. tForty-nine patients were not included due to missing SRF thickness data or a value of 0 (even though they had SRF at baseline).

BCVA, best-corrected visual acuity; MA, macular atrophy; SRF, subretinal fluid. are reported. For categorical variables, frequencies, percentages and $\chi^{2} \mathrm{p}$ values are reported. Observed data were used, and there was no imputation for missing data. All analyses were performed using SAS V.9.4.

\section{RESULTS}

\section{Baseline values}

A total of 785 ranibizumab-treated patients from the HARBOR trial with SRF at baseline (defined as screening, baseline or week 1) were included in this post hoc analysis. Key patient demographics and baseline characteristics are summarised in table 1. Baseline BCVA was evaluated as a function of SRF thickness at M12 or M24. Mean BCVA at baseline was greater in eyes with residual SRF at M12 compared with eyes with no SRF, regardless of SRF thickness (mean: no SRF, 53.6 (95\% CI 52.6 to 54.7) ETDRS letters; >0-50 $\mu \mathrm{m} \mathrm{SRF,}$ 58.7 (95\% CI 55 to 62.5) letters; > 50-100 $\mu \mathrm{m}$ SRF, 59.1 (95\% CI 56.8 to 61.5 ) letters; $>100 \mu \mathrm{m}$ SRF, 59.4 (95\% CI 56.5 to 62.4 ) letters). Similar trends were observed in mean BCVA at baseline and M24 SRF thickness.

\section{Effect of residual SRF on risk of developing MA following ranibizumab treatment}

Analysis based on FA and CFP

The impact of residual SRF thickness on developing MA after ranibizumab treatment was assessed in eyes with no MA at baseline $(n=676)$. The presence of treatmentresistant residual SRF at M3 was associated with significantly lower rates of MA at M12 and M24 compared with eyes with resolved SRF at M3. MA rate with residual versus resolved SRF at M3 was $5.1 \%$ vs $22.1 \%$, respectively, at M12 ( $p<0.0001$ ), and $13.3 \%$ vs $31.2 \%$, respectively, at M24 $(p<0.0001)$ (figure 1A). Similarly, in eyes with no MA at baseline, presence of treatment-resistant residual SRF at M6 was also associated with significantly lower rates of MA at M12 ( $<<0.0001)$ and M24 ( $<<0.0001)$ compared with eyes with resolved SRF at M6 (figure 1B). When eyes were placed in subgroups by thickness of SRF at baseline, a numerically higher proportion of those in the $>100 \mu \mathrm{m}$ SRF thickness group had no MA (vs those with MA) at M12 (68.9\% vs $61.9 \%$, respectively) and M24 (70.6\% vs $61.5 \%$, respectively), although these differences were not statistically significant.

\section{Analysis based on MA status from SD-OCT}

Using SD-OCT to identify MA, 652/785 (83.1\%) eyes had MA absent at baseline, and 92/785 (11.7\%) eyes had MA present. MA status was missing for $9(1.1 \%)$ and questionable for $32(4.1 \%)$ of 785 eyes at baseline. Among eyes with no MA at baseline, the rate of MA at M12 was $18.7 \%$ $(85 / 455)$ in eyes with no SRF at M3 and $8.1 \%(11 / 135)$ in eyes with residual SRF at M3 $(p=0.0036)$. By M24, the rate was $28.1 \%(126 / 448)$ among eyes with no SRF at M3 and $15.6 \%(21 / 135)$ among eyes with residual SRF at M3 $(p=0.0032)$ (figure $2 \mathrm{~A})$. Among eyes with no MA at baseline, the rate of MA at M12 was $18.3 \%(80 / 438)$ in eyes with no SRF at M6 and 9.6\% (15/156) in eyes with residual SRF at M6 $(\mathrm{p}=0.0114)$. By M24, the MA rate was $28.8 \%(124 / 431)$ among eyes with no SRF at M6 and 13.9\% (22/158) among eyes with residual SRF at M6 $(\mathrm{p}=0.0002)$ (figure $2 \mathrm{~B})$. Thus, the SD-OCT data corroborate the findings of the FA and CFP data regarding the rate of MA development in the presence and absence of treatment-resistant subretinal fluid (TR-SRF). 
A

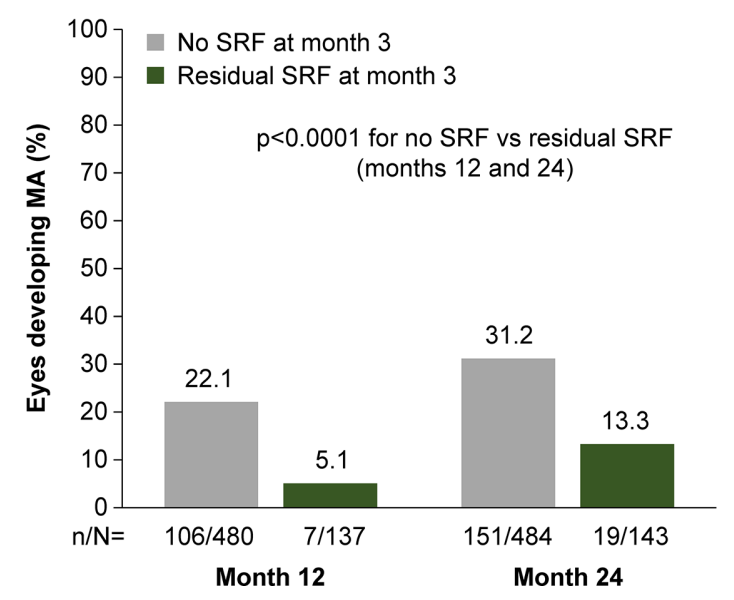

B

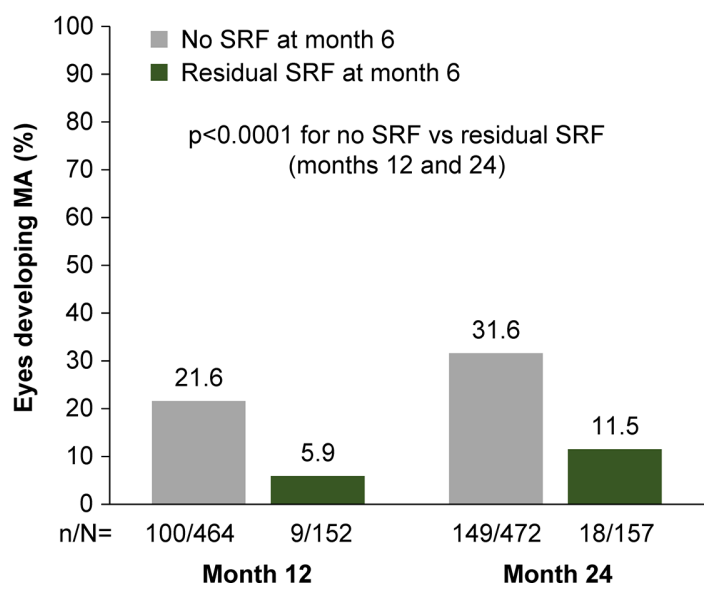

Figure 1 Effect of residual SRF on the rate of MA development in eyes with no MA at baseline. Rate of ranibizumab-treated eyes (with no MA at baseline) developing MA at months 12 and 24 is shown for eyes with no residual SRF versus those with residual SRF at (A) month 3 and (B) month 6. MA, macular atrophy; SRF, subretinal fluid.

\section{Effect of baseline SRFV on risk of developing MA following ranibizumab treatment}

To assess the impact of baseline SRFV on the risk of developing MA, we quantified SRFV centred under the fovea in areas with diameters of (1) $1 \mathrm{~mm}$, (2) $3 \mathrm{~mm}$ and (3) $6 \mathrm{~mm}$.

\section{Analysis based on FA and CFP}

Greater baseline SRFV was associated with lower rates of MA at M12 and M24 (figure 3A,B; online supplemental figure S2). SRFV at baseline, grouped into quartiles, was evenly distributed in eyes that did not develop MA by M12, whereas there was a trend towards lower baseline SRFV among eyes that developed MA by M12 (figure 3A). A similar trend was observed at M24 (figure $3 \mathrm{~B}$ ). Overall, there was no obvious effect of different circle diameters on the results.

\section{Analysis based on SD-OCT}

Among eyes with no MA at baseline, we found no significant difference in mean SRFV at baseline by MA status at M12 or M24 (online supplemental table S1). However, among eyes with no MA at baseline, eyes with SRFV in the first quartile, the smallest amount of SRF at baseline, had higher rates of MA at M12 compared with the other three quartiles, although the trend was not statistically significant for the $3-\mathrm{mm}$ circle $(p=0.0856)$ and was of borderline significance for the $6-\mathrm{mm}$ circle $(p=0.0419)$ (online supplemental figure S3A). A similar trend was seen at M24 (online supplemental figure S3B).

\section{Effect of residual SRF thickness on visual acuity following ranibizumab treatment}

To evaluate the impact of residual SRF thickness on visual acuity changes following ranibizumab treatment, BCVA was evaluated at M12 and M24 for study eyes grouped by SRF thickness. At M12, ranibizumab-treated eyes with residual SRF had greater mean BCVA compared with eyes that had no SRF, regardless of SRF thickness: no SRF, 63.6 ETDRS letters; $>0-50 \mu \mathrm{m}$ SRF, 71.2 letters; $>50-100 \mu \mathrm{m}$ SRF, 71.3 letters; and $>100 \mu \mathrm{m}$ SRF, 69.2 letters (figure 4A). These trends in BCVA values by SRF thickness were similar at M24 (figure 4B). When mean change in BCVA from baseline was considered, there were no differences
A

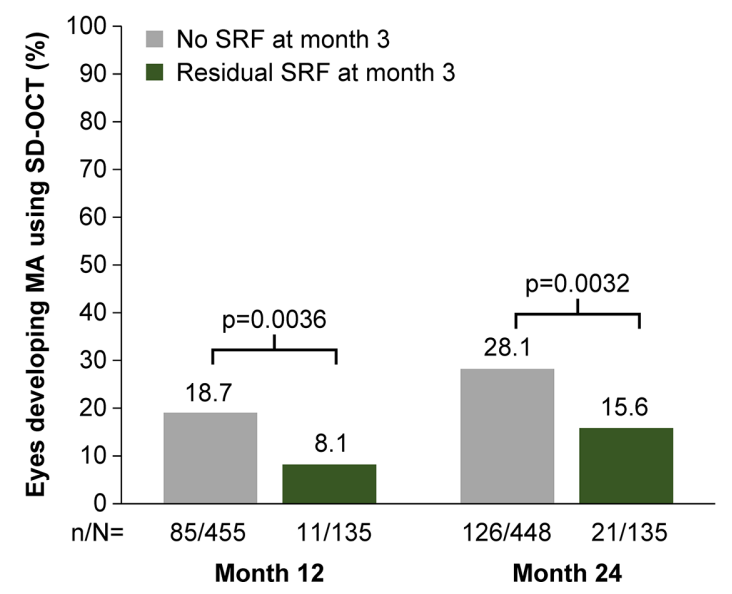

B

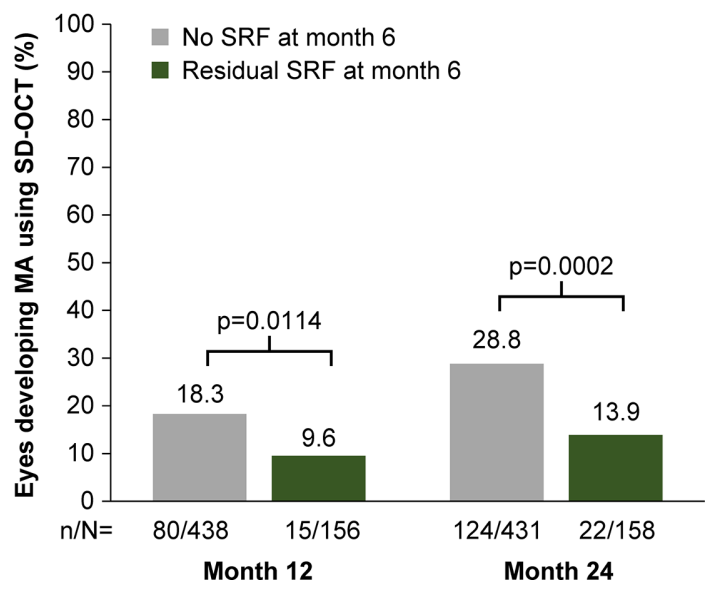

Figure 2 Effect of residual SRF on MA status from SD-OCT. Rate of ranibizumab-treated eyes (with no MA at baseline) developing MA at months 12 and 24 is shown for eyes with no residual SRF versus those with residual SRF at (A) month 3 and (B) month 6. MA, macular atrophy; OCT, optical coherence tomography; SRF, subretinal fluid. 
A

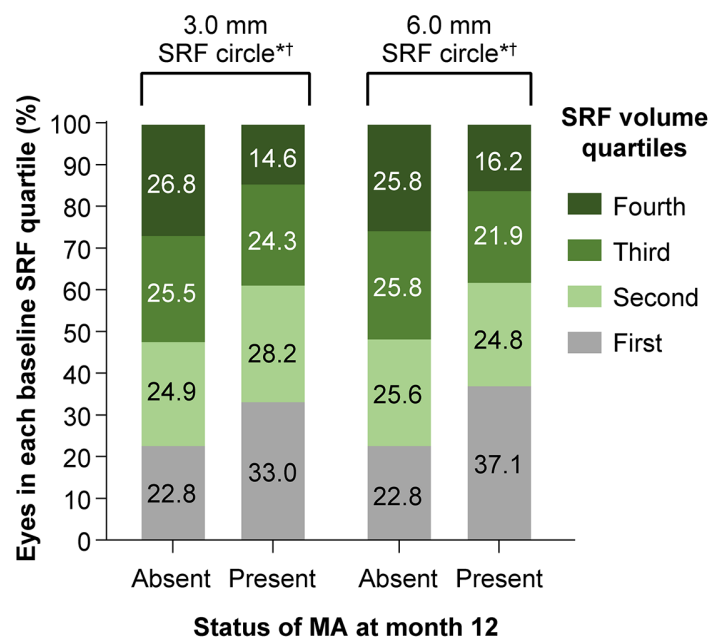

B

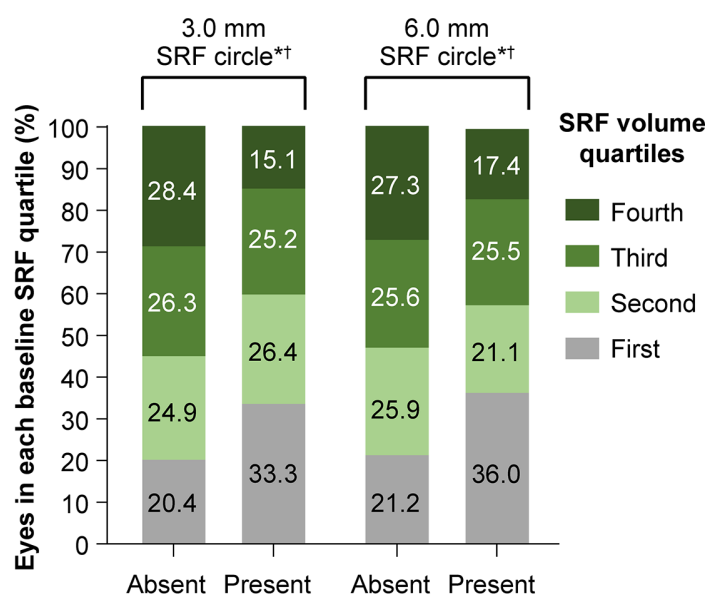

Status of MA at month 24

Figure 3 Baseline SRFV by quartile and MA at months 12 and 24. MA was identified using colour fundus photographs and fluorescein angiograms. First and fourth quartiles represent the lowest and highest SRFVs, respectively. There was a trend for SRFV at baseline to be larger in eyes without MA and smaller in eyes that developed MA by (A) month 12 or (B) month 24. The MA analysis was limited to study eyes with SRF of $>0$ at baseline, screening or week 1 and MA absent at baseline. SRFV that occurred more extensively in the area centralis was classified as 3-and 6-mm diameter around the estimated retinal centre. SRFV was not analysed in the area solely under the fovea (1.0-mm diameter around the retinal centre) because more than half of patients had volume $=0$ at baseline in this location. ${ }^{*} p<0.05$ for comparing the distribution of SRFV between the MA status groups. ${ }^{\dagger}$ Diameter of retina-centred circle used to measure baseline SRFV. MA, macular atrophy; SRF, subretinal fluid; SRFV, subretinal fluid volume.

between eyes with residual SRF and those with no SRF, regardless of SRF thickness, both at M12 and M24 (figure 4C,D).

\section{Effect of choroidal thickness and SRF thickness}

We found no meaningful correlation between SRF thickness and choroidal thickness at baseline or M24 (online supplemental table S2).

\section{DISCUSSION}

We analysed anti-VEGF-resistant SRF in $50-\mu \mathrm{m}$ increments and found a reduced risk of MA, regardless of SRF thickness, with no threshold detrimental to vision outcomes over 2 years. These findings were obtained regardless of whether MA was assessed using FA and CFPs or SD-OCT, despite the fact that SD-OCT is a superior method of assessing the presence of MA. (We note that fundus autofluorescence data are not available in the HARBOR dataset.) Among eyes with no MA at baseline, we found no significant difference in mean SRFV at baseline by MA status at M12 or M24, as judged by SD-OCT images. In contrast, results using CFP and FA to identify MA suggested that greater baseline SRFV was associated with lower rates of MA at M12 and M24. The discrepancy may be due to the difficulty in identifying MA in the presence of SRF. Overall, these findings are consistent with earlier analyses. ${ }^{6-8}$

Previously, Sadda $e \mathrm{al}^{7}$ showed that TR-SRF was associated with a lower incidence of MA in this cohort. In the present analysis, we sought to determine whether this relationship was biphasic. Although a small amount of SRF might not be detrimental, a large amount might be detrimental. Quantitatively, concentration (c) is a function of distance $(\mathrm{x})$ and time $(\mathrm{t})$, as well as the diffusion constant (D) and total mass (M). The relation is $c(x, t)=M(4 \pi D t)^{-1 / 2} \exp \left(-x^{2} /(4 D t)\right.$. Thus, diffusion of essential components would be expected to decrease exponentially as a function of distance. Examining the effect of increasing amounts of SRF should allow a robust determination of the impact of SRFV on the incidence of MA. Based on these considerations, one might expect increased amounts of SRF to be associated with worse visual outcomes. However, the opposite was the case, and increased TR-SRF and, less consistently, increased SRFV at baseline, were both associated with a lower risk of MA. Eyes with TR-SRF exhibited comparable levels of change in BCVA compared with eyes without TR-SRF. We also note that patients with TR-SRF (vs those with no SRF) at M12 and M24 had greater baseline BCVA, as well as higher SRFV at baseline. These facts prompt a reconsideration of the biological significance of SRF in nAMD.

Hypoxia is the main physiological signal for expression of VEGF-A. ${ }^{15}$ When solid tumours grow, the rapidly dividing cells are gradually displaced from the existing capillaries. ${ }^{16}$ The resulting hypoxia leads to VEGF-A expression, and new capillaries sprout to meet the oxygen demands of the growing tumour. ${ }^{17}$ One hypothesis of age-related macular degeneration (AMD) pathogenesis is that damage to the RPE and choriocapillaris is associated with outer retinal hypoxia. ${ }^{18-21}$ Hypoxia triggers VEGF expression and CNV formation. ${ }^{22}$ In the absence of an adequate homeostatic $\mathrm{CNV}$ response, MA ensues. Persistent SRF in the course of anti-VEGF treatment may be a sign of persistent $\mathrm{CNV}$ perfusion with transudation, which may operate as an imperfect compensatory mechanism that maintains the function of the degenerating macula. This suggestion is consistent with what has been observed clinically, ${ }^{23-27}$ as well as in a post hoc analysis of SD-OCT data derived from the HARBOR trial. ${ }^{14}$ The permeability properties of CNV, either innately in some patients or induced by anti-VEGF therapy in others, may alter the biochemical composition of the SRF so that it more closely mimics the extracellular milieu to which the photoreceptors are normally exposed. Not all SRFs are the same, as demonstrated by the different effects of SRF associated with idiopathic central serous chorioretinopathy ${ }^{28}$ and macula-involving rhegmatogenous retinal detachment ${ }^{29}$ on visual acuity. Therefore, the SRF could potentially be a manifestation of an imperfect yet critical 'survival mechanism' for the degenerating macula. The cessation or absence of this imperfect survival mechanism may be a harbinger of the development of atrophic changes (online 
A

Mean BCVA at month 12

by SRF thickness at month 12

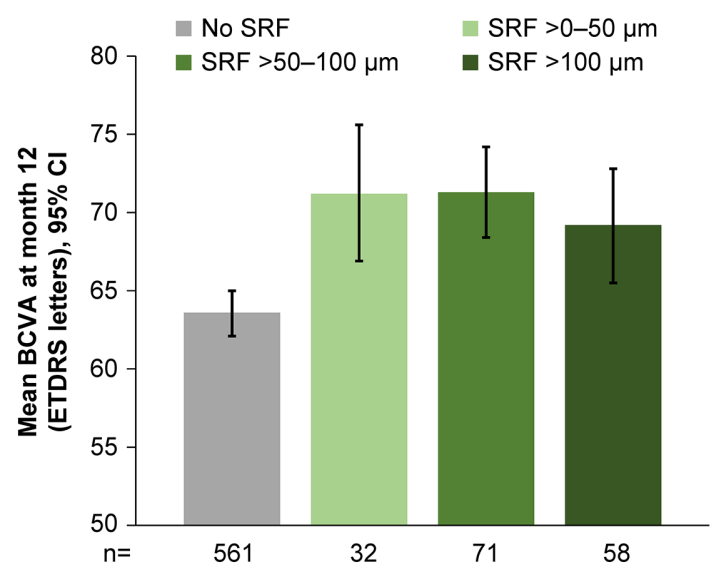

C

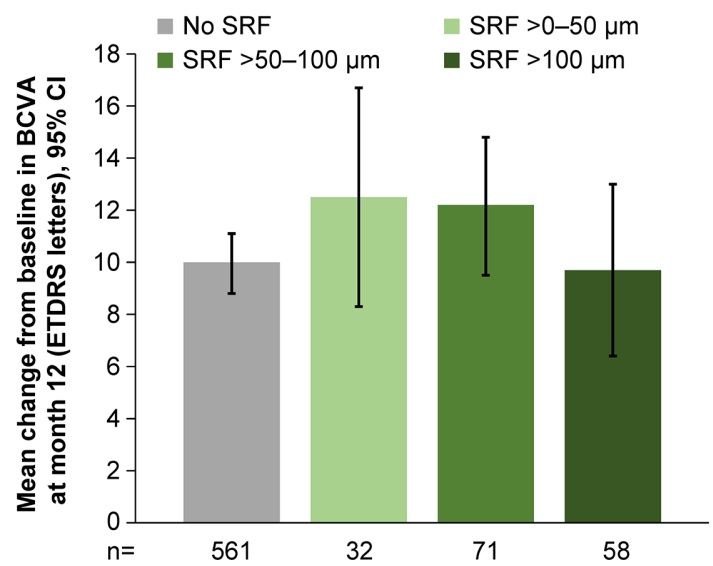

Mean change in BCVA at month 12 by SRF thickness at month 12
B

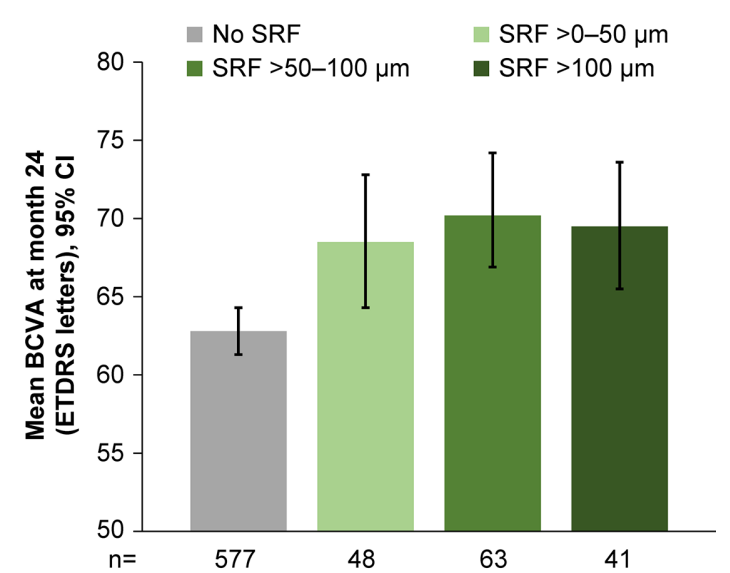

D

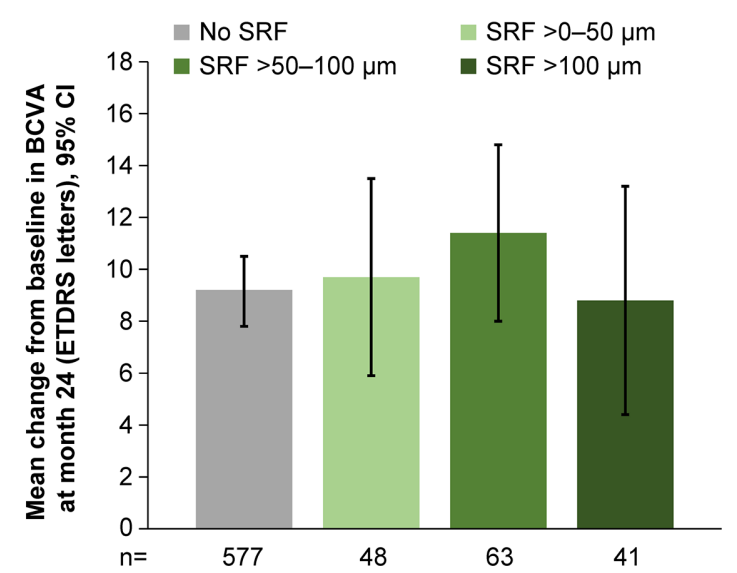

Mean change in BCVA at month 24 by SRF thickness at month 24
63

41

Figure 4 Mean BCVA at months 12 and 24 by SRF thickness and mean change from baseline in BCVA at months 12 and 24 by SRF thickness. Mean BCVA is shown for ranibizumab-treated eyes grouped by SRF thickness at (A) month 12 and (B) month 24. Mean change from baseline to (C) month 12 and (D) month 24 is shown for eyes grouped by SRF thickness. Error bars represent 95\% Cls. BCVA, best-corrected visual acuity; SRF, subretinal fluid.

supplemental figure S4). From this post hoc analysis, we postulate that the higher baseline BCVA observed in patients who were identified as having residual SRF later in the study may be an indication that this protective mechanism was already in place at baseline. In this regard, we note that Sadda $e$ t $a l^{14}$ reported that a $100-\mu \mathrm{m}$ increase in baseline central subfoveal choroidal thickness was associated with a $\sim 25 \%$ decreased risk of any MA or definite MA. Thus, patients with thinner choroids had an increased risk of MA. This finding may indicate that patients with lower choroidal perfusion capacity are at increased risk, and that $\mathrm{CNV}$ is a homeostatic response to AMD-associated choroidal damage.

Change in BCVA was similar among cohorts that had no SRF versus some SRF, despite the difference in incidence of MA. Given the relatively brief duration of follow-up (2 years), the impact of MA on visual outcomes is difficult to determine, as has also been observed by other investigators. ${ }^{30} 31$ Although the posology of the HARBOR and FLUID ${ }^{8}$ trials differed, the visual acuity results are similar in that persistent SRF of $<200 \mu \mathrm{m}$ was not associated with a worse visual acuity outcome.

We emphasise that the data presented here are derived from a post hoc analysis of clinical trial results. The correlation of reduced risk of MA associated with the presence of
SRF does not per se constitute a rationale for treating CNV less aggressively with anti-VEGF agents. All of these patients were treated aggressively with anti-VEGF therapy, which suggests that this is anti-VEGF-resistant SRF. The presence of SRF in the setting of anti-VEGF therapy might simply identify a subset of patients with better anatomical outcomes for other, more fundamental reasons than simply the presence of residual SRF. For example, the presence of TR-SRF might be related to a difference in the molecular architecture of the choroidal new vessels in these patients or might reflect a difference in the degree of AMD-induced choroidal damage. Alternatively, the presence of TR-SRF might indicate the presence of relatively greater amounts of healthy tissue (eg, RPE-choroid) capable of producing VEGF as well as maintaining photoreceptor function. In other words, TR-SRF might be a biomarker of some other aspect of the patient's anatomy or physiology that favours a reduced risk of MA development. Eliminating patients with IRF from this analysis may have biased the sample towards patients with better retinal structural integrity and hence a lower risk of MA. Nonetheless, it is important to recognise that residual SRF in the course of anti-VEGF treatment may be a predictor of positive treatment outcomes, at least with regard to the evolution of MA. 
Contributors MAZ participated in the data analysis and preparation of the manuscript and figures. LH contributed to the study design, data analysis and manuscript and figure preparation. AM and MG provided the deep learning algorithm used to generate the volumetric data and helped to interpret the results. IS contributed to the study design and interpretation as well as the manuscript preparation. All authors reviewed, contributed to and approved the manuscript and take responsibility for its content.

Funding Funding was provided by Genentech, Inc., a member of the Roche Group, for the study. Genentech, Inc. participated in the design of the HARBOR trial. Medical reviewers from Genentech, Inc., were allowed to review this manuscript for accurate reporting of the data but did not make decisions regarding the final interpretation of the data or overall content of this paper. Funding was provided by Genentech, Inc., for third-party writing assistance, which was provided by Ray Beck, Jr, PhD, and Michelle Kelly, PhD, of Envision Pharma Group.

Competing interests MAZ: consultant: Boehringer Ingelheim, Foundation Fighting Blindness, Frequency Therapeutics, Genentech, Inc./Roche, Iduna, Iveric Bio, Life Biosciences, Novartis Pharma AG, Ophthotech, Perfuse Therapeutics, Selphagy; equity owner: NVasc; financial support, patent: Rutgers University. LH: consultant: Aerpio, Alimera, Genentech, Inc., PolyPhotonix, Recens Medical. AM and MG: employee, equity owner: F. Hoffmann-La Roche Ltd. IS: employee, equity owner: Genentech, Inc.

Patient consent for publication Obtained.

Ethics approval The HARBOR clinical trial adhered to the tenets of the Declaration of Helsinki. Independent ethics committees or institutional review boards approved HARBOR, and all patients provided written informed consent.

Provenance and peer review Not commissioned; externally peer reviewed.

Data availability statement Data may be obtained from a third party and are not publicly available. Qualified researchers may request access to individual patientlevel data through the clinical study data request platform (https://vivli.org/). Further details on Roche's criteria for eligible studies are available online (https://vivli.org/ members/ourmembers/). Further details on Roche's Global Policy on the Sharing of Clinical Information and how to request access to related clinical study documents are available online (https://www.roche.com/research_and_development/who_we_ are_how_we_work/clinical_trials/our_commitment_to_data_sharing.htm).

Supplemental material This content has been supplied by the author(s). It has not been vetted by BMJ Publishing Group Limited (BMJ) and may not have been peer-reviewed. Any opinions or recommendations discussed are solely those of the author(s) and are not endorsed by BMJ. BMJ disclaims all liability and responsibility arising from any reliance placed on the content. Where the content includes any translated material, BMJ does not warrant the accuracy and reliability of the translations (including but not limited to local regulations, clinical guidelines, terminology, drug names and drug dosages), and is not responsible for any error and/or omissions arising from translation and adaptation or otherwise.

Open access This is an open access article distributed in accordance with the Creative Commons Attribution Non Commercial (CC BY-NC 4.0) license, which permits others to distribute, remix, adapt, build upon this work non-commercially, and license their derivative works on different terms, provided the original work is properly cited, appropriate credit is given, any changes made indicated, and the use is non-commercial. See: http://creativecommons.org/licenses/by-nc/4.0/.

\section{ORCID iD}

Marco A Zarbin http://orcid.org/0000-0002-7811-7132

\section{REFERENCES}

1 Brown DM, Kaiser PK, Michels M, et al. Ranibizumab versus verteporfin for neovascular age-related macular degeneration. N Engl J Med 2006;355:1432-44.

2 Rosenfeld PJ, Brown DM, Heier JS, et al. Ranibizumab for neovascular age-related macular degeneration. N Engl J Med 2006;355:1419-31.

3 Lalwani GA, Rosenfeld PJ, Fung AE, et al. A variable-dosing regimen with intravitreal ranibizumab for neovascular age-related macular degeneration: year 2 of the PrONTO study. Am J Ophthalmol 2009;148:43-58.

4 Engelbert M, Zweifel SA, Freund KB. Long-term follow-up for type 1 (subretinal pigment epithelium) neovascularization using a modified "treat and extend" dosing regimen of intravitreal antivascular endothelial growth factor therapy. Retina 2010;30:1368-75.

5 Freund KB, Korobelnik J-F, Devenyi R, et al. Treat-and-extend regimens with anti-VEGF agents in retinal diseases: a literature review and consensus recommendations. Retina 2015;35:1489-506.
6 Grunwald JE, Pistilli M, Daniel E, et al. Incidence and growth of geographic atrophy during 5 years of Comparison of Age-related Macular Degeneration Treatments Trials. Ophthalmology 2017;124:97-104

7 Sadda SR, Tuomi LL, Ding B, et al. Macular atrophy in the HARBOR study for neovascular age-related macular degeneration. Ophthalmology 2018;125:878-86.

8 Guymer RH, Markey CM, McAllister IL, et al. Tolerating subretinal fluid in neovascular age-related macular degeneration treated with ranibizumab using a treat-and-extend regimen: FLUID study 24-month results. Ophthalmology 2019;126:723-34.

9 Sadda SR, Ghanekar A, Hill L. Relationship between sub retinal fluid (SRF) and vision outcomes in eyes with neovascular age-related macular degeneration (nAMD) in the HARBOR trial. Invest Ophthalmol Vis Sci 2019;60:5189.

10 Holekamp NM, Ghanekar A, Hill L. What do the HARBOR data reveal about the relationship between the drying of subretinal and intraretinal fluid and associated vision outcomes?37th Annual Meeting of the American Society of Retina Specialists; 26-30 July, Chicago, IL, 2019.

11 Busbee BG, Ho AC, Brown DM, et al. Twelve-month efficacy and safety of $0.5 \mathrm{mg}$ or $2.0 \mathrm{mg}$ ranibizumab in patients with subfoveal neovascular age-related macular degeneration. Ophthalmology 2013;120:1046-56.

12 Ho AC, Busbee BG, Regillo CD, et al. Twenty-four-month efficacy and safety of $0.5 \mathrm{mg}$ or $2.0 \mathrm{mg}$ ranibizumab in patients with subfoveal neovascular age-related macular degeneration. Ophthalmology 2014;121:2181-92.

13 Ronneberger O, Fischer P, Brox T. U-Net: convolutional networks for biomedical image segmentationMedical Image Computing and Computer-Assisted Intervention MICCAI 2015. , 2015: 9351, 234-41. doi:10.1007/978-3-319-24574-4_28

14 Sadda SR, Abdelfattah NS, Lei J, et al. Spectral-domain OCT analysis of risk factors for macular atrophy development in the HARBOR study for neovascular age-related macular degeneration. Ophthalmology 2020;127:1360-70.

15 Shweiki D, Itin A, Soffer D, et al. Vascular endothelial growth factor induced by hypoxia may mediate hypoxia-initiated angiogenesis. Nature 1992;359:843-5.

16 Jain RK, Martin JD, Stylianopoulos T. The role of mechanical forces in tumor growth and therapy. Annu Rev Biomed Eng 2014;16:321-46.

17 Ferrara N, Adamis AP. Ten years of anti-vascular endothelial growth factor therapy. Nat Rev Drug Discov 2016;15:385-403.

18 Sarks JP, Sarks SH, Killingsworth MC. Morphology of early choroidal neovascularisation in age-related macular degeneration: correlation with activity. Eye 1997; 11:515-22

19 McLeod DS, Lutty GA. High-resolution histologic analysis of the human choroidal vasculature. Invest Ophthalmo/ Vis Sci 1994;35:3799-811.

20 Grossniklaus HE, Green WR. Choroidal neovascularization. Am J Ophthalmol 2004; 137:496-503.

21 Mullins RF, Dewald AD, Streb LM, et al. Elevated membrane attack complex in human choroid with high risk complement factor $\mathrm{H}$ genotypes. Exp Eye Res 2011;93:565-7.

22 Miller JW, Le Couter J, Strauss EC, et al. Vascular endothelial growth factor A in intraocular vascular disease. Ophthalmology 2013;120:106-14.

23 Querques G, Srour M, Massamba N, et al. Functional characterization and multimodal imaging of treatment-naive "quiescent" choroidal neovascularization. Invest Ophthalmol Vis Sci 2013;54:6886-92.

24 Dansingani KK, Freund KB. Optical coherence tomography angiography reveals mature, tangled vascular networks in eyes with neovascular age-related macular degeneration showing resistance to geographic atrophy. Ophthalmic Surg Lasers Imaging Retina 2015;46:907-12.

25 Dhrami-Gavazi E, Balaratnasingam C, Lee W, et al. Type 1 neovascularization may confer resistance to geographic atrophy amongst eyes treated for neovascular agerelated macular degeneration. Int J Retina Vitreous 2015;1:15.

26 Capuano V, Miere A, Querques L, et al. Treatment-naïve quiescent choroidal neovascularization in geographic atrophy secondary to nonexudative age-related macular degeneration. Am J Ophthalmol 2017;182:45-55.

27 Christenbury JG, Phasukkijwatana N, Gilani F, et al. Progression of macular atrophy in eyes with type 1 neovascularization and age-related macular degeneration receiving long-term intravitreal anti-vascular endothelial growth factor therapy: an optical coherence tomographic angiography analysis. Retina 2018;38:1276-88.

28 Nicholson B, Noble J, Forooghian F, et al. Central serous chorioretinopathy: update on pathophysiology and treatment. Surv Ophthalmol 2013;58:103-26.

29 Burton TC. Recovery of visual acuity after retinal detachment involving the macula. Trans Am Ophthalmol Soc 1982;80:475-97.

30 Chakravarthy U, Harding SP, Rogers CA, et al. Alternative treatments to inhibit VEGF in age-related choroidal neovascularisation: 2-year findings of the IVAN randomised controlled trial. Lancet 2013;382:1258-67.

31 Martin DF, Maguire MG, Fine SL, et al. Ranibizumab and bevacizumab for treatment of neovascular age-related macular degeneration: two-year results. Ophthalmology 2012;119:1388-98. 